\title{
El método del caso en la didáctica de la Geografía
}

\author{
Ricard Gay féraiz y Juan José Buil Callén
}

\section{INTRODUCCIÓN AL MÉdOTO DEL CASO. SU IMPORTANCIA}

Partiendo de la base que la metodologia participativa tiene como finalidad crear una situación óptima de trabajo - situación de aprendizaje - para lograr determinados objetivos de mejora personal y que dentro de las técnicas que facilitan la participación destaca el método del caso, consideramos que podría ser de gran ayuda introducir dicha técnica participativa en el aula para los alumnos de $8 .^{\circ}$ de EGB y los tres cursos de BUP y FP.

Antes de seguir adelante debemos hacer algunas precisiones sobre el método del caso.

Un caso es la descripción de una situación real, generalmente compleja, en la que existen problemas, es decir, situaciones a mejorar, relacionadas con los temas que son objeto de estudio para un determinado grupo de trabajo ${ }^{1}$.

Pone a disposición de los participantes todo tipo de datos -importantes y secundarios; problemáticos y anecdóticos - referentes a una realidad a mejorar.

En cualquiera de sus modalidades - breve o largo; secuencial o no; con anexos o sin ellos, etc.- un caso sirve para analizar una situación.

' O. F. Otero, El método del caso. ICE Universidad de Navarra, 1978. 
El método del caso es una técnica de metodología participativa orientada al trabajo en equipo, a partir del trabajo personal -del profesor y de los alumnos-.

Por lo tanto, ya vemos que no se trata de olvidar el trabajo individual, del esfuerzo personal; no, no se trata de eso sino de todo lo contrario.

En la discusión dirigida de un caso se pretende aunar la aportación de ideas de varias personas -en este caso de los alumnos- en torno a un tema o a un bloque de problemas.

La dirección de esta discusión ha de conseguir relacionar y sintetizar estas ideas, en orden a unos objetivos de aprendizaje, sin menoscabo de la riqueza de matices de este trabajo en equipo, y del respeto a cada alumno.

Las aportaciones personales, implican, al menos, coherencia entre to que pensamos y decimos, y lo que hacemos o haríamos.

El análisis de un caso comporta la oportunidad y la disposición de participar responsablemente. El método del caso, correctamente utilizado es altamente motivador ya que supone la participación de todos y cada uno de los alumnos.

Cada alumno se debe esforzar para expresarse sintéticamente y brevemente; en saber escuchar - ese difícil arte hoy en día- y en saber responder; en distinguir lo que es objetivo de discusión y lo que no lo es.

El método del caso obliga al alumno a diagnosticar y definir los problemas; a establecer un orden de importancia entre ellos, de modo que podamos otorgar prioridades en la búsqueda de soluciones.

La importancia del método del caso radica, como bien dice el profesor Llano, en ser «un método de enseñanza que discute con el alumno lo que ha de hacerse frente a situaciones concretas; es, justamente, el de pretender el desarrollo de la prudencia mas que la comunicación (sea por exposición, sea por descubrimiento o vivencia personal) del conocimiento o saber científico".

\section{OBJETIVOS QUE SE PERSIGUEN Y APRENDIZAJES}

Teniendo en cuenta que los rasgos psicológicos característicos de las edades de los alumnos a los que va dirigida esta experiencia son 
entre otros: a) la originalidad, la autodisciplina, la curiosidad cientifica y el trabajo en equipo a nivel de aprendizaje; b) la reflexión, deducción, la generalización, la síntesis, la introspección y el criterio lógico a nivel de pensamiento; c) y la inestabilidad a nivel de intereses, hemos establecido los siguientes objetivos:

A. Fomentar la mejora personal mediante la relación humana (trabajo en equipo) (EGB, BUP, FP):

A.1. Adquirir actitud positiva con respecto a las personas y a las ideas (EBG, BUP, FP).

A.2. Dar posibles soluciones a los problemas planteados (EGB, BUP, FP).

B. Pasar de un comportamiento más o menos pasivo a un comportamiento activo, de participante.

B.1. Saber hablar:

B.1.1. Emplear el léxico apropiado (EGB, BUP, FP).

B.1.2. Intervenir oportunamente (BUP, FP).

B.2. Saber escuchar (Respetar el turno de palabra, estar callados...) (EGB, BUP, FP).

B.3. Fomentar el espiritu participativo (EGB, BUP, FP).

B.4. Establecer una prioridad en los problemas (BUP, FP).

B.5. Abstraer de los hechos posibles consecuencias (EGB, BUP, FP, cada uno a su nivel).

B.6. Enjuiciar los hechos (EGB, BUP, FP).

C. Ayudar a captar mejor la realidad:

C.1. Adquisición de los conocimientos propios del tema (EGB, BUP, FP).

C.2. Enfocar el tema desde distintos ángulos, aportando conocimientos desde los diversos saberes humanos.

Sabiendo que un caso se elige en función de unos objetivos relacionados con un área de estudio, el aprendizaje consistiría en perfeccionar modelos de decisión significativos para resolver determinados problemas concretos. 
Sin embargo, mas que otra cosa, la enseñanza a través del método del caso pretende que el estudiante se acostumbre a pensar en cuestiones de fondo a partir de situaciones concretas. Por eso, debe estar relacionado con otras sesiones - previas o posteriores- en las que se procura asimilar una información sintética, sistemática de un tema.

No se trata de que el estudiante aprenda datos - para eso existen otros documentos de trabajo menos costosos en tiempo-; se trata de que esos conocimientos que posee de otras ciencias o de sus vivencias personales los interrelacione y los use en ese preciso momento. No pretende enseñar verdades sino fomentar capacidades.

\section{DOCUMENTOS DE APOYO}

Entre la gran variedad de documentos complementarios que pueden utilizarse (series estadísticas, gráficas, mapas, textos alusivos al tema, recortes de prensa, etc...), merece especial atención para trabajar con los alumnos de estas edades la "Nota Técnica» - especialmente en los cursos superiores de BUP y FP- y la «Ficha Técnica» para el resto.

La Nota Técnica y la Ficha Técnica las elabora el profesor o el equipo de profesores que colaboran interdisciplinariamente en la experiencia. Estas no tienen por qué estar redactadas en función de los objetivos del caso en cuestión, aunque sí deben hacer referencia a objetivos colaterales.

¿Qué es una Nota Técnica? Simplemente un documento con un máximo de información y un mínimo de cuestiones para reflexionar sobre ellas, al contrario que la Ficha Técnica que es un máximo de preguntas y un mínimo de información.

\section{UN EJEMPLO APLICADO}

«En un principio, quienes integran un grupo de aprendizaje, en el que se introduce la metodología participativa — concretamente el método 
del caso-, necesita aprender a participar" ${ }^{2}$. Por tanto, hay que informar previamente de la mecánica del caso. Dicha mecánica consiste en una lectura previa individual del caso, seguida de una reunión de trabajo en pequeño grupo con el fin de fomentar la participación. A continuación en una reunión de gran grupo se van exponiendo los hechos que aportan los alumnos de los diferentes grupos. El profesor los anota en la pizarra insistiendo en no confundir hechos con opiniones, y facilita una visión global de lo anotado en la pizarra. De estos hechos se deducen los problemas y se anotan en el encerado para buscar posibles soluciones.

Este podría ser el desarrollo de un caso:

A) Lectura individual del caso.

B) Reunión por grupos de cuatro o cinco alumnos para discutir el caso con el objeto de:

1. Anotar los hechos (Hay que poner especial atención en no confundir hechos con opiniones subjetivas fruto de una lectura imparcial o subjetiva del texto).

2. Destacar los problemas que aparecen en el caso.

C) Se anota en la pizarra los hechos que aportan los diferentes grupos.

D) A la luz de los hechos señalados en la pizarra se indican los posibles problemas que los diferentes grupos han creído ver en el caso (Conviene hacer una sintesis de tal modo que los problemas queden reducidos a dos como máximo).

E) Se proponen soluciones individualmente y se discute su viabilidad.

En algunas ocasiones las soluciones que se proponen dan pie a una discusión amplia que se puede canalizar en un debate, con un guión que pueden elaborar los alumnos, con la ayuda del profesor, y preparar con el material estadístico y bibliográfico pertinente, para que se hable con razón de causa en el debate. Éste, puede ser moderado por un alumno que, al finalizar, sintetiza las intervenciones de sus compañeros y concluye el debate. Dichas conclusiones sirven para retornar por última vez al caso y revisar la viabiliada y la coherencia lógica de algunas de las soluciones que se propusieron al caso.

2 J. J. BulL, E. GARCiA, Aproximación metodológica a la formación de orientadores familiares. Barcelona. Memoria de Lic. 1980. 
El caso que presentamos ha estado trabajado en múltiples ocasiones por diferentes alumnos de diversas edades. Algunos de los hechos señalados, entre otros muchos y sólo a modo de ejemplo, han sido: - China es el pais más poblado del mundo. -El gobierno quiere estabilizar la población en 1.200.000.000 de personas. - Su densidad de población es de 93 habitantes por $\mathrm{km}^{2}$. - El $66 \%$ del suelo chino alberga tan sólo al $11 \%$ de la población, mientras que las regiones del Este tienen una densidad de casi $300 \mathrm{hab} . / \mathrm{km}^{2}$. -El gobierno está aplicando y desarrollando campañas de control demográfico, etc...

Entre los problemas que más veces se ha hecho mención hay que destacar los siguientes: La superpoblación en China, la superpoblación tan sólo en las regiones del Este, la gran tasa de natalidad previa a las campañas de control de la natalidad estatal, etc.

Los debates se han centrado casi en su totalidad en torno a la superpoblación. El título de uno de ellos fue: "Superpoblación en el mundo: ¿Realidad o falso alarmismo?».

\section{EL PAPEL DEL PROFESOR}

El método del caso es un buen instrumento en manos de un profesor experimentado. "Pero si bien el análisis de un caso requiere experiencia, también es verdad que la genera: la resolución de un caso sirve para experimentar la experiencia, y no sólo para adquirirla de oídas» ${ }^{3}$.

La resolución de un caso genera experiencia a pesar de ser una «experiencia simulada" más o menos bien aprovechada por el profesor para el desarrollo de capacidades; entre ellas, debe destacarse la capacidad de analogía.

Por otra parte, no todo profesor es adecuado para utilizar el método del caso como técnica didáctica. Se precisa que sea una persona que sepa ayudar a descubrir diversas soluciones a un problema, sin prescindir nunca de algunos principios, pocos y muy generales: punto de partida,

${ }^{3}$ C. LLANO, "El método del caso y el desarrollo de capacidades activas", en Revista EAFIT, Medellín, Colombia 1978. 
luces orientadoras de la acción, jamás metas cómodas o conclusiones que indiquen un exclusivo modo de hacer en cada caso.

La actuación de quien dirige o modera un caso es siempre difícil. En efecto, requiere una tremenda disciplina por su parte. No solamente debe renunciar a imponer su propia personalidad, sino que ha de mostrar, con un ejemplo, que respeta a los participantes y estima sus puntos de vista.

¿De qué depende el éxito del profesor que dirige el caso? Pues del nivel alcanzado en una serie de capacidades en desarrollo; de sus propias actitudes: confianza, respeto, comprensión y exigencia; de la adecuación del propio caso escrito; del conocimiento de sí mismo y del grupo de alumnos cuyo trabajo orienta; de la cantidad y calidad de la participación conseguida.

\section{RESULTADOS OBSERVADOS}

Se ha conseguido una mayor cohesión en las clases.

Ante un problema determinado los alumnos piensan según varias alternativas en vez de en una sola.

Proponen seguir con el sistema del método del caso en otras clases, porque para los de EGB es más divertido y, en cambio, los alumnos de BUP y FP se sienten más protagonistas en la materia. En definitiva, la motivación en ambos grupos es mayor.

Los alumnos mayores sugieren cómo se podría haber evitado el problema antes de llegar a la situación descrita en el caso.

Los de EGB se entretienen mucho más tiempo en el análisis de hechos que en el de problemas y por supuesto de soluciones.

Los alumnos siguen hablando del caso durante bastante tiempo, tras finalizar su estudio, e incluso preguntan a otros profesores cómo lo hubieran solucionado ellos.

Facilita mejor la comprensión y asimilación de los temas en los que se ha aplicado esta técnica didáctica.

Conforme se va aplicando, se observa un mayor respeto hacia las opiniones ajenas. 
Hemos observado una gran influencia de los medios de comunicación social con «slogans" y campañas publicitarias sobre los juicios que emiten los alumnos al analizar una situación.

Los razonamientos de los alumnos son en principio unilaterales, no objetivos, debidos precisamente a la machaconería de esos medios, en particular TV.

\section{UN CASO}

\section{El control de la natalidad en la República Popular China}

China es el país más poblado del mundo (algo más de mil millones en el último censo). El gobierno está aplicando enérgicas medidas para reducir la tasa de natalidad y estabilizar la cifra de población en 1.200 millones.

Es el tercer país en extensión del mundo con una densidad de $93 \mathrm{~h} /$ $\mathrm{km}^{2}$. Las provincias del oeste y del norte cuentan entre uno y diez habitantes por $\mathrm{km}^{2}$. El conjunto de estas provincias cubre aproximadamente la mitad del territorio chino, y es una gran extensión para el trabajo de rozadores y pioneros, jun Far West todavía por explotar! Un segundo grupo de provincias presenta densidades débiles -de 36 a 60-. Las posibilidades de asentamiento humano son todavía muy amplias, las condiciones naturales menos apremiantes y los costes sociales de ordenación menos importantes que en las primeras provincias. Estos dos primeros conjuntos cubren $6.380 .000 \mathrm{~km}^{2}$; es decir, el $66 \%$ del suelo chino agrupa al $11 \%$ de la población. El conjunto siguiente está representado por la China oriental, que en una superficie de $2.240 .000 \mathrm{~km}^{2}$, es decir el $23,5 \%$ del suelo, agrupa el $76 \%$ de la población, con una densidad media de $297 \mathrm{~h} / \mathrm{km}^{2}$.

Entre 1949 y 1980 la tasa de natalidad se redujo a la mitad, una caída sin precedentes en un gran país en vías de desarrollo. En 1949 era de 35 nacimientos anuales por cada 1.000 habitantes, nivel que se había mantenido durante muchas décadas. A consecuencia de la supresión de la propiedad privada sobre las tierras de cultivo y de las primeras campañas de control de la natalidad, dicha tasa descendió a 25 por mil a finales de la década de 1950. Durante la Revolución Cultural y otros avatares políticos de los años sesenta la planificación de la natalidad dejó 
de constituir un objetivo oficial y la tasa de natalidad aumentó rápidamente hasta un máximo del 44 por mil anual.

En la década de 1970 empezó a manifestarse plenamente el efecto de un control demográfico organizado. La Tercera Campaña de Planificación de los Nacimientos, con el lema "Más tarde, más largo, menos", propugnaba el matrimonio a edad más tardía, un intervalo más largo entre embarazos y un menor número de hijos. En 1979 le siguió un programa todavía más enérgico: La Campaña del Hijo Único. Al entrar en 1980 , la tasa de natalidad habia descendido al 18 por mil. Las cifras oficiales quizá hayan subestimado el número de nacimientos, y recientemente se ha producido también un incremento de la fertilidad. En consecuencia, en estos momentos la tasa de natalidad podría situarse alrededor del 20 por mil.

La eficacia de las recientes campañas de control de natalidad se debe principalmente a la estructura política de la sociedad china. Esta estructura es el legado de los 20 años de lucha por el poder que libraron Mao y sus seguidores. En la lucha se seleccionaban cuidadosamente los cuadros, que se preparaban en escuelas secretas del partido; cada cuadro se convertía entonces en un eslabón de la estructura unificada de mando. Cuando el Partido Comunista tomó el poder, la mayor conciencia y organización política desarrolladas durante la guerra se encauzaron hacia fines civiles, entre ellos la planificación familiar. Los servicios de control de la natalidad están integrados dentro del sistema sanitario y cada organismo administrativo cuenta con personas especializadas en la educación para la planificación familiar. Pequeños grupos de discusión dirigidos por cuadros del partido se encargan de transmitir el concepto de control de la población desde las más altas instancias ejecutivas hasta las parejas casadas.

Además de ofrecer argumentos en favor del control demográfico, el aparato administrativo se encarga de distribuir material para el control de la natalidad, así como recompensas y castigos. Se ofrecen gratuitamente píldoras anticonceptivas, dispositivos intrauterinos (DIU) y operaciones de esterilización para las parejas casadas, así como exámenes físicos prematrimoniales que comprenden explicaciones sobre la anticoncepción.

Los procedimientos que se siguen para convencer a las parejas casadas de que limiten su fecundidad se dejan a la discreción de las autoridades locales, pero en todas las localidades se ofrecen recompensas para seguir la planificación familiar y se castiga a quienes tienen demasiados hijos. Se pide a las parejas de recién casados que firmen una declaración en la que se comprometen a tener un solo hijo. Hasta media- 
dos de 1981 más de once millones de matrimonios habían firmado ese acuerdo, que les da derecho a recibir atención hospitalaria gratuita durante el parto, atención médica y educación gratuita para la criatura, prioridad en la concesión de una vivienda mejor y una paga mensual extra al año. Los hijos únicos, y sobre todo las hijas únicas, gozan de un trato preferente en el colegio y en la obtención de empleo.

Los castigos impuestos por los embarazos no aprobados pueden ser considerables. El nacimiento de una segunda criatura de un matrimonio que haya firmado el documento de hijo único da lugar a una amonestación oficial. El nacimiento de un tercer hijo se castiga con una reducción del salario. Muchas mujeres que quedan encinta sin permiso son inducidas a abortar. Por abortar se premia a la mujer con unas vacaciones pagadas, y lo mismo ocurre si se somete a una operación de esterilización con fines anticonceptivos. La ejecución descentralizada de las decisiones políticas, a cargo de celosos cuadros locales del Partido que compiten por hacer méritos, facilitan la coacción, tanto en el caso del aborto como en el de la esterilización.

Los datos para la redacción del presente caso han sido tomados de:

1. Gentelle, P., 1977, La China. Ed. Ariel, colección Elcano.

2. KEYFITZ, N., 1984, «La población china». Revista Investigación y Ciencia. 